\title{
Cancellation of Multiuser Interference Due to Carrier Frequency Offsets in Uplink OFDMA
}

\author{
Shamaiah Manohar, Dheeraj Sreedhar, Vibhor Tikiya, and A. Chockalingam, Senior Member, IEEE
}

\begin{abstract}
In uplink orthogonal frequency division multiple access (OFDMA) systems, multiuser interference (MUI) occurs due to different carrier frequency offsets (CFO) of different users at the receiver. In this paper, we present a multistage linear parallel interference cancellation (LPIC) approach to mitigate the effect of this MUI in uplink OFDMA. The proposed scheme first performs CFO compensation (in time-domain) followed by $K$ DFT operations (where $K$ is the number of users) and multistage LPIC on these DFT outputs. We scale the MUI estimates by weights before cancellation and optimize these weights by maximizing the average signal-to-interference ratio (SIR) at the output of the different stages of the LPIC. We derive closed-form expressions for these optimum weights. The proposed LPIC scheme is shown to effectively cancel the MUI caused by the other user CFOs in uplink OFDMA. While our proposed approach performs CFO compensation in time-domain, an alternate approach proposed recently by Huang and Letaief performs CFO compensation and interference cancellation in frequency-domain. We show that our approach performs better than the Huang \& Letaief's approach when the magnitude of the CFO differences (between desired user CFO and other user CFOs) are small, whereas their approach performs better when the magnitude of the individual CFOs (of other users) are small. Since the CFO values can be arbitrary at the receiver, in order to make the receiver robust under various CFO conditions, we propose simple metrics based on CFO knowledge, which the receiver can compute and use to choose between the time-domain (ours) and the frequency-domain (Huang \& Letaief's) cancellers so that better performance among the two approaches is achieved under various CFO conditions.
\end{abstract}

Index Terms - Carrier frequency offset, circular convolution, linear parallel interference cancellation, optimum weights, signalto-interference ratio, uplink OFDMA.

\section{INTRODUCTION}

$\mathbf{R}$ ECENT research has been witnessing increased focus on orthogonal frequency multiple access (OFDMA) on the uplink [1]-[10]. The performance of OFDM/OFDMA systems

Manuscript received November 16, 2005; revised July 28, 2006; accepted September 15,2006 . The associate editor coordinating the review of this paper and approving it for publication was V. Lau. This work in part was presented in the IEEE Wireless Communications and Networking Conference, Las Vegas, April 2006, and in the International Conference on Communications, Istanbul, June 2006. This work was supported in part by the Swarnajayanti Fellowship, Department of Science and Technology, New Delhi, Government of India, under Project Ref: No.6/3/2002-S.F, and the DRDO-IISc Program on Advanced Research in Mathematical Engineering.

S. Manohar is with Honeywell Technology Solutions Lab Private Limited, Bangalore 560076, India (e-mail: manohar.shamaiah@honeywell.com).

D. Sreedhar is with Sasken Communication Technologies Limited, Bangalore 560071, India (e-mail: dheeraj_sreedhar@yahoo.com).

V. Tikiya is with the Indian Institute of Management, Ahmedabad, India (e-mail: vibhor_tikiya@yahoo.com).

A. Chockalingam is with the Department of Electrical Communication Engineering, Indian Institute of Science, Bangalore 560012, India (e-mail: achockal@ece.iisc.ernet.in).

Digital Object Identifier 10.1109/TWC.2007.05905 depend to a large extent on how well the orthogonality among different subcarriers are maintained at the receiver [11],[12]. Factors including carrier frequency offsets (CFO) between the transmitter and receiver induced by Doppler effects and/or poor oscillator alignments, sampling clock frequency discrepancies, and time delay caused by multipath and nonideal synchronization can destroy the orthogonality among subcarriers. Among the above factors, the impact of CFO on the performance is the most crucial one because the CFO values are large (typically of the order of several $\mathrm{KHz}$ ) due to carrier frequencies being of the order of $\mathrm{GHz}$. In uplink OFDMA, correction to one user's CFO would misalign other initially aligned users. Thus, other user CFO will result in significant multiuser interference (MUI) in uplink OFDMA.

There have been a few recent attempts in the literature that address the issue of MUI due to other user CFO in uplink OFDMA [7]-[10]. The approach proposed in [7] is to feedback the estimated CFO values to the mobiles so that the mobile transmitters can adjust their transmit frequencies. This needs additional signaling and hence reduces system throughput. An alternate approach is to apply interference cancellation (IC) techniques at the base station (BS) receiver [8]-[10]. Recently, in [9], Huang and Letaief presented an IC approach which performs CFO compensation and MUI cancellation in frequency-domain using circular convolution. We refer to this scheme in [9] as Huang-Letaief Circular Convolution (HLCC) scheme. The circular convolution approach was proposed earlier by Choi et al in [6] as an alternative to the direct timedomain method of CFO compensation. Huang and Letaief refer the scheme in [6] as CLJL scheme (CLJL stands for the first letters of the names of the four authors of [6]). The CLJL scheme does not perform MUI cancellation. The HLCC scheme uses circular convolution for both CFO compensation (as in [6]) as well as MUI cancellation. In [10], we have proposed a minimum mean square error (MMSE) receiver for MUI cancellation in uplink OFDMA. We derived a recursion to approach the MMSE solution and showed that this recursive MMSE solution encompasses the CLJL and HLCC schemes as special cases.

Structure-wise, a common feature in CLJL [6], HLCC [9], and MMSE [10] schemes is that all these detectors/cancellers first perform a single DFT operation on the received samples and the resulting DFT output vector is further processed to achieve CFO compensation and MUI cancellation using circular convolution. A new contribution in this paper is that we propose and analyze an alternate MUI cancellation receiver structure which first performs CFO compensation in timedomain, followed by $K$ DFT operations (where $K$ is the 
number of users) and multistage linear parallel interference cancellation (LPIC) on these DFT outputs. We scale the estimated MUI by weights before cancellation. For this proposed scheme, we derive closed-form expressions for the average signal-to-interference ratio (SIR) at the output of various stages of the LPIC. We also derive closed-form expressions for the optimum weights that maximize the average SIR at the output of the different LPIC stages.

We make interesting observations on the performance and complexity comparison between the proposed WLPIC scheme (employing time-domain approach) and the HLCC scheme in [9] (employing frequency-domain approach). In terms of performance, we observe that in the WLPIC scheme the bit error performance is affected by the magnitude of the $\mathrm{CFO}$ differences (between the desired user $\mathrm{CFO}$ and the other user CFOs), whereas in the HLCC scheme the performance is affected by the magnitude of the individual CFOs (of other users). Because of this, the WLPIC scheme performs better than the HLCC scheme when the magnitude of the CFO differences are small, whereas the HLCC scheme performs better when the magnitude of the individual CFOs are small. The CFO values at the receiver can be arbitrary in practice. So, in order to make the receiver robust under various CFO conditions, we propose simple metrics based on CFO knowledge, which the receiver can compute and use to choose between the WLPIC (ours) and the HLCC (Huang \& Letaief's) schemes so that better performance among the two approaches is achieved under various CFO conditions. In terms of complexity, we show that the proposed WLPIC scheme is less complex than the HLCC scheme, particularly when the number of subcarriers is large (which is typical in OFDMA systems).

The rest of this paper is organized as follows. In Section II, we present the uplink OFDMA system model. The proposed WLPIC scheme is presented in Section III. Section IV provides the SIR analysis of the proposed scheme. The SIR and bit error rate (BER) performance results and performance/complexity comparison with HLCC scheme are presented in Section V. Conclusions are given in Section VI.

\section{SySTEM MODEL}

We consider an uplink OFDMA system with $K$ users, where each user communicates with a base station through an independent multipath channel as shown in Fig. 1. We assume that there are $N$ subcarriers in each OFDM symbol and one subcarrier can be allocated to only one user. The information symbol for the $i$ th user on the $k$ th subcarrier is denoted by $X_{k}^{(i)}, k \in S_{i}$, where $S_{i}$ is the set of subcarriers assigned to user $i$ and $E\left[\left|X_{k}^{(i)}\right|^{2}\right]=1$. Then, $\bigcup_{i=1}^{K} S_{i}=\{0,1, \ldots, N-1\}$ and $S_{i} \bigcap S_{j}=\phi$, for $i \neq j$. The length of the guard interval added is $N_{g}$ samples and is assumed to be longer than the maximum channel delay spread. After IDFT processing and guard interval insertion at the transmitter, the time-domain sequence of the $i$ th user, $x_{n}^{(i)}$, is given by

$$
x_{n}^{(i)}=\frac{1}{N} \sum_{k \in S_{i}} X_{k}^{(i)} e^{\frac{j 2 \pi n k}{N}}, \quad-N_{g} \leq n \leq N-1 .
$$

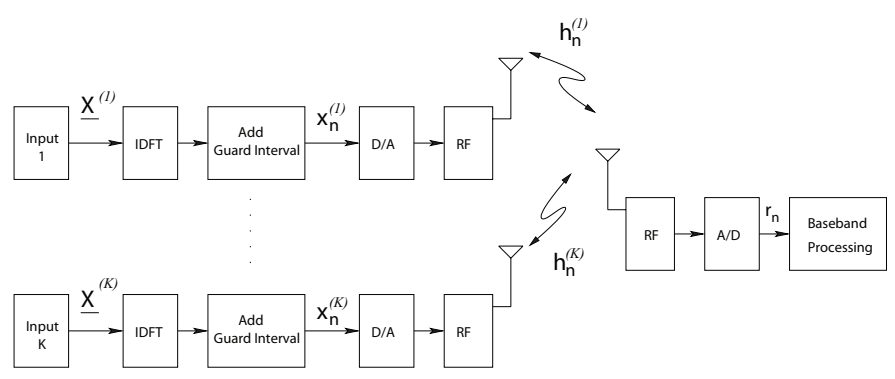

Fig. 1. Uplink OFDMA system model.

The $i$ th user's signal at the receiver input, after passing through the channel, is given by

$$
s_{n}^{(i)}=x_{n}^{(i)} \star h_{n}^{(i)},
$$

where $\star$ denotes linear convolution and $h_{n}^{(i)}$ is the $i$ th user's channel impulse response. It is assumed that $h_{n}^{(i)}$ is non-zero only for $n=0, \ldots, L-1$, where $L$ is the maximum channel delay spread, and that all users' channels are statistically independent. We assume that $h_{n}^{(i)}$ 's are i.i.d. complex Gaussian with zero mean and $E\left[\left(h_{n, I}^{(i)}\right)^{2}\right]=E\left[\left(h_{n, Q}^{(i)}\right)^{2}\right]=1 / 2 L$, where $h_{n, I}^{(i)}$ and $h_{n, Q}^{(i)}$ are the real and imaginary parts of $h_{n}^{(i)}$. The channel coefficient in frequency-domain $H_{k}^{(i)}$ is given by

$$
H_{k}^{(i)}=\sum_{n=0}^{L-1} h_{n}^{(i)} e^{\frac{-j 2 \pi n k}{N}}
$$

and $E\left[\left|H_{k}^{(i)}\right|^{2}\right]=1$. The received baseband signal after coarse carrier frequency tracking (leaving some residual carrier frequency offset) is given by

$$
r_{n}=\sum_{i=1}^{K} s_{n}^{(i)} e^{\frac{j 2 \pi \epsilon_{i} n}{N}}+z_{n}, \quad-N_{g} \leq n \leq N-1,
$$

where $\epsilon_{i}, i=1, \ldots, K$ denotes $i$ th user's residual carrier frequency offset (CFO) normalized by the subcarrier spacing, and $z_{n}$ is the AWGN with zero mean and variance $\sigma^{2}$. We assume that all users are time synchronized and that $\epsilon_{i}, i=$ $1, \cdots, K$ are known at the receiver.

Fig. 2 shows the receiver baseband processing including $i$ ) CFO compensation in time-domain and guard time removal, ii) $K$ DFT operations (one for each user), and iii) linear parallel interference cancellation (LPIC) in multiple stages. Note that the CFO compensation is carried out in time-domain by multiplying $r_{n}$ with $e^{-\frac{j 2 \pi \epsilon_{i} n}{N}}, i=1, \cdots, K$ (this method of CFO compensation is referred to as the direct method in [6]). The received signal after CFO compensation and guard time removal for the $i$ th user is given by

$$
y_{n}^{(i)}=r_{n} e^{-\frac{j 2 \pi \epsilon_{i} n}{N}}, \quad 0 \leq n \leq N-1,
$$

which forms the input to the $i$ th DFT block. The output of the DFT block for the $i$ th user on the $k$ th subcarrier is then given by

$$
Y_{k}^{(i)}=\underbrace{H_{k}^{(i)} X_{k}^{(i)}}_{\text {Desired signal }}+\underbrace{\sum_{\substack{l=1 \\ l \neq i}}^{K} \sum_{q \in S_{l}} \rho_{k q}^{(i),(l)} H_{q}^{(l)} X_{q}^{(l)}}_{\text {MUI }}+\underbrace{Z_{k}^{(i)}}_{\text {Noise }}
$$


where

$$
\rho_{k q}^{(i),(l)}=\frac{\sin \pi\left(k-q+\delta_{l i}\right)}{N \sin \frac{\pi}{N}\left(k-q+\delta_{l i}\right)} e^{-j\left(1-\frac{1}{N}\right) \pi\left(k-q+\delta_{l i}\right)},
$$

and $\delta_{l i}$ is the difference between the $i$ th user and $l$ th user CFO values given by

$$
\delta_{l i}=\epsilon_{l}-\epsilon_{i}
$$

The channel coefficient $H_{k}^{(i)}$ is given by (3) and the noise component $Z_{k}^{(i)}$ is given by

$$
Z_{k}^{(i)}=\sum_{n=0}^{N-1} z_{n}^{(i)} e^{\frac{-j 2 \pi n\left(k+\epsilon_{i}\right)}{N}} .
$$

Note that the 2nd term in (6) represents the CFO-induced MUI present at the DFT output. In the case of single user detection (SUD), the DFT outputs, $Y_{k}^{(i)}$, s, can be directly used to make the symbol decision. Additional processing may be performed on $Y_{k}^{(i)}$ 's in order to mitigate the effect of MUI. For example, multistage interference cancellation techniques can be employed to improve performance. In the next section, we propose a multistage weighted linear parallel interference cancellation scheme which operates on the DFT outputs, $Y_{k}^{(i)}$ 's.

\section{Proposed Weighted Linear PIC Scheme}

It is noted that, for the desired user $i$, the 2 nd term in (6) represents the CFO-induced MUI (i.e., interference from other users $l=1,2, \cdots, K, l \neq i$ ) present at the DFT output. Also, from Eqns. (6),(7),(8), it can be noted that the amount of this MUI depends on $\delta_{l i}$ 's, the differences between desired user CFO $\left(\epsilon_{i}\right)$ and other user CFOs $\left(\epsilon_{l}\right.$ 's). Our aim is to cancel this 2nd term in (6) using a multistage linear PIC approach, where an estimate of the MUI in a given stage is obtained using the soft values of previous stage outputs (without any non-linear operation, e.g., hard decision, on the previous stage outputs $)^{1}$. Further, it is known that the MUI estimates in an LPIC approach can become quite inaccurate under poor channel conditions (e.g., low SNR, high interference) to such an extent that it may be better not to do cancellation [14]. Such situations can be alleviated by scaling the MUI estimates by weights (preferably by some optimum weights) before cancellation [17],[18]. Here, we present such a weighted LPIC (WLPIC) scheme for the uplink OFDMA.

The proposed multistage WLPIC scheme is shown in Fig. 2. Let $m$ denote the stage index. We take the DFT outputs, $Y_{k}^{(i)}$,s, in (6) as the first stage $(m=1)$ outputs of the receiver, i.e., $Y_{k,(1)}^{(i)}=Y_{k}^{(i)}$. In the case of SUD, the symbol decisions are made directly from $Y_{k,(1)}^{(i)}$,s. LPIC is performed in the subsequent stages. In a given LPIC stage $m, m>1$, an estimate of the MUI is made based on the soft values of the

\footnotetext{
${ }^{1}$ Alternatively, MUI estimates can be obtained using hard estimates of the data symbols, $\widehat{X}_{q}^{l}$, s, obtained using hard decision on previous stage outputs. This results in a non-linear PIC which is analytically less tractable. The multistage PIC originally proposed by Varanasi and Aazhang in [13] and several other PICs in the literature for CDMA belong to this type of nonlinear PIC. Here, we consider a linear PIC approach, which is also widely studied in CDMA systems [14]-[18]. Linear PIC approach is attractive because of its analytical tractability, implementation simplicity and good performance [14],[16],[18].
}

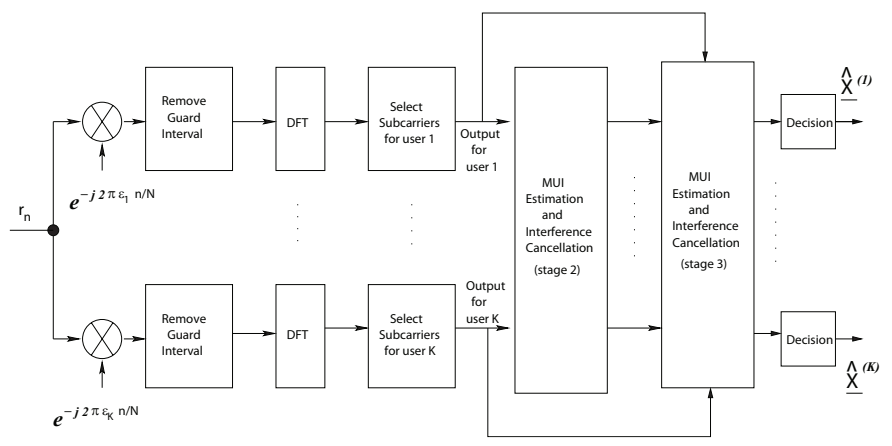

Fig. 2. Receiver baseband processing - CFO compensation and multistage interference cancellation.

previous stage outputs. These MUI estimates are scaled by weights and subtracted (i.e., cancelled) from the DFT outputs, $Y_{k,(1)}^{(i)}$.

As we mentioned earlier, we seek to cancel the 2 nd term in (6). Towards that end, consider the following operation on the other user DFT outputs, $Y_{q,(1)}^{(l)}$ :

$$
\sum_{\substack{l=1 \\ l \neq i}}^{K} \sum_{q \in S_{l}} \rho_{k q}^{(i),(l)} Y_{q,(1)}^{(l)}
$$

Using (6) in the above, we can write

$$
\begin{aligned}
& \sum_{\substack{l=1 \\
l \neq i}}^{K} \sum_{q \in S_{l}} \rho_{k q}^{(i),(l)} Y_{q,(1)}^{(l)}=\sum_{\substack{l=1 \\
l \neq i}}^{K} \sum_{q \in S_{l}} \rho_{k q}^{(i),(l)}\left(H_{q}^{(l)} X_{q}^{(l)}\right. \\
& \left.+\sum_{\substack{j=1 \\
j \neq l}}^{K} \sum_{r \in S_{j}} \rho_{q r}^{(l),(j)} H_{r}^{(j)} X_{r}^{(j)}+Z_{q}^{(l)}\right) \\
& =\underbrace{\sum_{\substack{l=1 \\
l \neq i}}^{K} \sum_{q \in S_{l}} \rho_{k q}^{(i),(l)} H_{q}^{(l)} X_{q}^{(l)}}_{T_{1}} \\
& +\underbrace{\sum_{\substack{l=1 \\
l \neq i}}^{K} \sum_{q \in S_{l}} \rho_{k q}^{(i),(l)} \sum_{\substack{j=1 \\
j \neq l}}^{K} \sum_{r \in S_{j}} \rho_{q r}^{(l),(j)} H_{r}^{(j)} X_{r}^{(j)}}_{T_{2}} \\
& +\underbrace{\sum_{\substack{l=1 \\
l \neq i}}^{K} \sum_{q \in S_{l}} \rho_{k q}^{(i),(l)} Z_{q}^{(l)}}_{T_{3}} .
\end{aligned}
$$

Note that the 1 st term $T_{1}$ in (11) is the same as the MUI term (i.e., the 2 nd term) in (6), which we wanted to cancel. Hence, (10) can be viewed as an MUI estimate for the 2nd stage of the LPIC, which when cancelled (i.e., subtracted) from (6) will completely remove the MUI term (i.e., the 2 nd term) in (6). In the process, additional terms $T_{2}$ (interference) and $T_{3}$ (noise), which were not there in the 1 st stage output, get introduced in the 2 nd stage output. The interference term $T_{2}$ introduced in the 2nd stage can be cancelled in the 3rd stage using a similar MUI estimate obtained from $Y_{q,(2)}^{(l)}$ 's, and so on. Accordingly, in the proposed WLPIC, the interference cancelled output of the $i$ th user on the $k$ th subcarrier in the $m$ th stage, $Y_{k,(m)}^{(i)}$, 
$m>1$, can be written as

$$
Y_{k,(m)}^{(i)}=Y_{k,(1)}^{(i)}-w_{k,(m)}^{(i)} \underbrace{\sum_{\substack{l=1 \\ l \neq i}}^{K} \sum_{q \in S_{l}} \rho_{k q}^{(i),(l)} Y_{q,(m-1)}^{(l)}}_{\text {MUI estimate for } m \text { th stage }},
$$

where $Y_{k,(1)}^{(i)}$ is the 1 st stage output given by $(6), \rho_{k q}^{(i),(l)}$ is given by (7), and $w_{k,(m)}^{(i)}$ is the weight with which the MUI estimate is scaled. We call the WLPIC scheme with the weights on all subcarriers of all users to be unity (i.e., $\left.w_{k,(m)}^{(i)}=1, \forall i, k, m\right)$ as conventional LPIC (CLPIC) scheme. In the CLPIC scheme, the operations needed for the choice of optimum weights and MUI scaling with these weights are avoided (because of unity weights). However, performance better than that of the CLPIC can be achieved by using optimum weights. We propose to obtain the optimum weights for the $m$ th stage, $w_{k,(m)}^{(i), o p t}, i=1,2, \cdots, K, k \in S_{i}$, by maximizing the corresponding average SIR at the $m$ th stage output.

In an uncoded system, the symbol decision for the $i$ th user on the $k$ th subcarrier at the output of the $m$ th stage can be made based on the output $Y_{k,(m)}^{(i)}$. For example, the symbol decision at the $m$ th stage output for the case of BPSK modulation can be obtained as

$$
\widehat{X}_{k,(m)}^{(i)}=\operatorname{sgn}\left(\mathcal{R} e\left(\overline{H_{k}^{(i)}} Y_{k,(m)}^{(i)}\right)\right) .
$$

In the above equation and henceforth, we use 'overline' to denote conjugate operation, i.e., $\overline{H_{k}^{(i)}}$ denotes the conjugate of $H_{k}^{(i)}$. For the case of $M$-QAM/M-PSK modulation, symbol decision can be made using the minimum Euclidean distance rule. In a coded system, the $Y_{k,(m)}^{(i)}$ 's are fed to the decoder.

\section{SIR ANALYSIS}

In this section, we derive expressions for the average SIR at the output of the 2nd and 3rd stages of the proposed weighted LPIC scheme. Also, we will use the derived average SIR expressions to obtain closed-form expressions for the optimum weights $w_{k,(m)}^{(i), o p t}$.

It is noted that the average output SIR on a given subcarrier will depend on several things including number of users, channel impulse response, number of subcarriers, CFO values, and type of subcarrier allocation. Here, we consider two types of subcarrier allocation, namely, $i$ ) block allocation and ii) interleaved allocation. In block allocation, a consecutive block of subcarriers is alloted to one user, the next block to another user, and so on. In interleaved allocation, the subcarriers of each user are uniformly interleaved with the subcarriers assigned to the other users.

\section{A. Average SIR at the 2nd Stage Output}

From (12), the weighted interference cancelled output of the 2 nd stage $(m=2)$ for the $i$ th user on the $k$ th subcarrier is given by

$$
\begin{aligned}
Y_{k,(2)}^{(i)}= & H_{k}^{(i)} X_{k}^{(i)}\left(1-w_{k,(2)}^{(i)} \sum_{\substack{l=1 \\
l \neq i}}^{K} \sum_{q \in S_{l}} \rho_{k q}^{(i),(l)} \rho_{q k}^{(l),(i)}\right) \\
& +I_{2}+N_{2},
\end{aligned}
$$

where

$$
\begin{aligned}
I_{2}= & \sum_{l=1}^{K} \sum_{\substack{p \in S_{l} \\
p \neq k}} H_{p}^{(l)} X_{p}^{(l)}\left(\rho_{k p}^{(i),(l)}\left(1-w_{k,(2)}^{(i)}\right)\right. \\
& \left.-w_{k,(2)}^{(i)} \sum_{\substack{u=1 \\
u \neq i, l}}^{K} \sum_{v \in S_{u}} \rho_{k v}^{(i),(u)} \rho_{v p}^{(u),(l)}\right) \\
N_{2}= & Z_{k}^{(i)}-w_{k,(2)}^{(i)} \sum_{\substack{l=1 \\
l \neq i}}^{K} \sum_{q \in S_{l}} \rho_{k q}^{(i),(l)} Z_{l}^{(l)}
\end{aligned}
$$

The terms $I_{2}$ and $N_{2}$ in (15) and (16) represent the interference and noise terms introduced due to imperfect cancellation in using the soft output values from the first stage. After coherent combining using $\overline{H_{k}^{(i)}}$, the final output is given by

$$
\begin{aligned}
\overline{H_{k}^{(i)}} Y_{k,(2)}^{(i)}= & \left|H_{k}^{(i)}\right|^{2} X_{k}^{(i)}\left(1-w_{k,(2)}^{(i)} \sum_{\substack{l=1 \\
l \neq i}}^{K} \sum_{q \in S_{l}} \rho_{k q}^{(i),(l)} \rho_{q k}^{(l),(i)}\right) \\
& +I_{2}^{\prime}+N_{2}^{\prime},
\end{aligned}
$$

where $I_{2}^{\prime}=\overline{H_{k}^{(i)}} I_{2}$ and $N_{2}^{\prime}=\overline{H_{k}^{(i)}} N_{2}$.

Considering the $\overline{H_{k}^{(i)}} H_{p}^{(l)}$ factor in $I_{2}^{\prime}$, it is noted that the channel coefficients on subcarriers of different users $i 1$ and $i 2$, $H_{k 1}^{(i 1)}$ and $H_{k 2}^{(i 2)}, k 1 \in S_{i 1}, k 2 \in S_{i 2}, i 1 \neq i 2$, are uncorrelated because all users' channels are assumed to be independent. However, from (3), it can be seen that the channel coefficients on different subcarriers of the same user $i, H_{k 1}^{(i)}$ and $H_{k 2}^{(i)}$, $k 1, k 2 \in S_{i}$, are correlated. Also, this correlation depends on the subcarrier allocation. Handling the correlation between $H_{k 1}^{(i)}$ and $H_{k 2}^{(i)}$ in the SIR analysis is tedious. Therefore, to simplify the analysis, we assume that $H_{k 1}^{(i)}$ and $H_{k 2}^{(i)}$ are uncorrelated. Accordingly, the variance of $I_{2}^{\prime}, \sigma_{I_{2}^{\prime}}^{2}$, can be obtained as

$$
\sigma_{I_{2}^{\prime}}^{2}=\left|H_{k}^{(i)}\right|^{2} \sigma_{I_{2}}^{2}
$$

where

$$
\begin{aligned}
\sigma_{I_{2}}^{2}= & \sum_{l=1}^{K} \sum_{\substack{p \in S_{l} \\
p \neq k}} \mid \rho_{k p}^{(i),(l)}\left(1-w_{k,(2)}^{(i)}\right) \\
& -\left.w_{k,(2)}^{(i)} \sum_{\substack{u=1 \\
u \neq i, l}}^{K} \sum_{v \in S_{u}} \rho_{k v}^{(i),(u)} \rho_{v p}^{(u),(l)}\right|^{2},
\end{aligned}
$$

and the variance of $N_{2}^{\prime}, \sigma_{N_{2}^{\prime}}^{2}$, can be obtained as

$$
\sigma_{N_{2}^{\prime}}^{2}=\left|H_{k}^{(i)}\right|^{2} \sigma_{N_{2}}^{2},
$$

where

$$
\begin{aligned}
& \sigma_{N_{2}}^{2}=\sigma^{2}\left(N+2 N\left(w_{k,(2)}^{(i)}\right)^{2} \sum_{\substack{l=1 \\
l \neq i}}^{K} \sum_{q \in S_{l}}\left|\rho_{k q}^{(i),(l)}\right|^{2}+\right. \\
& \left(w_{k,(2)}^{(i)}\right)^{2} \sum_{\substack{l=1 \\
l \neq i}}^{K} \sum_{q \in S_{l}} \sum_{\substack{u=1 \\
u \neq i, l}}^{K} \sum_{v \in S_{u}} \rho_{k q}^{(i),(l)} \overline{\rho_{k v}^{(i),(u)}} \eta_{q v}^{(l),(u)} \\
& \left.-\mathcal{R} e\left[2 w_{k,(2)}^{(i)} \sum_{\substack{l=1 \\
l \neq i}}^{K} \sum_{q \in S_{l}} \rho_{k q}^{(i),(l)} \eta_{q k}^{(l),(i)}\right]\right)
\end{aligned}
$$


and

$$
\eta_{q k}^{(l),(i)}=E\left[Z_{q}^{(l)} \overline{Z_{k}^{(i)}}\right]=\sum_{n=0}^{N-1} e^{\frac{-j 2 \pi n\left(\epsilon_{l}-\epsilon_{i}+k-q\right)}{N}} .
$$

The average SIR on the $k$ th subcarrier of the $i$ th user at the output of the 2 nd stage, $S I R_{k,(2)}^{(i)}$, can then be obtained as

$$
S I R_{k,(2)}^{(i)}=\frac{\left(1-w_{k,(2)}^{(i)} \sum_{\substack{l=1 \\ l \neq i}}^{K} \sum_{q \in S_{l}} \rho_{k q}^{(i),(l)} \rho_{q k}^{(l),(i)}\right)^{2}}{\sigma_{I_{2}^{\prime}}^{2}+\sigma_{N_{2}^{\prime}}^{2}} .
$$

\section{B. Average SIR at the 3rd Stage output}

The soft values of the interference cancelled 2nd stage outputs of different users, $Y_{q}^{(l)}, l \neq i, q \in S_{l}$, are used to reconstruct (estimate) the MUI on the $k$ th subcarrier of the desired user $i$ in the 3 rd stage. These MUI estimates are then scaled by $w_{k,(3)}^{(i)}$ and cancelled. Accordingly, the weighted interference cancelled output of the 3rd stage for the $i$ th user on the $k$ th subcarrier, $Y_{k,(3)}^{(i)}$, is given by

$$
Y_{k,(3)}^{(i)}=H_{k}^{(i)} X_{k}^{(i)} F+I_{3}+N_{3}
$$

where

$$
\begin{aligned}
& F= 1-w_{k,(3)}^{(i)} \sum_{\substack{l=1, l \neq i}}^{K} \sum_{q \in S_{l}} \rho_{k q}^{(i),(l)}\left(\rho_{q k}^{(l),(i)}\left(1-w_{q,(2)}^{(l)}\right)\right. \\
&\left.-w_{q,(2)}^{(l)} \sum_{\substack{u=1 \\
u \neq l, i}}^{K} \sum_{v \in S_{u}} \rho_{q v}^{(l),(u)} \rho_{v k}^{(u),(i)}\right), \\
& I_{3}= \sum_{l=1}^{K} \sum_{\substack{q \in S_{l} \\
q \neq k}} H_{q}^{(l)} X_{q}^{(l)} \rho_{k q}^{(i),(l)} \\
& \cdot\left(1-w_{k,(3)}^{(i)}\left(1-w_{q,(2)}^{(l)} \sum_{\substack{u=1 \\
u \neq l}}^{K} \sum_{v \in S_{u}} \rho_{q v}^{(l),(u)} \rho_{v q}^{(u),(l)}\right)\right) \\
&-w_{k,(3)}^{(i)} \sum_{\substack{u=1 \\
u \neq i, l}}^{K} \sum_{v \in S_{u}} \rho_{k v}^{(l),(u)}\left(\rho_{v q}^{(u),(l)}\left(1-w_{v,(2)}^{(u)}\right)\right. \\
&\left.\left.-w_{v,(2)}^{(u)} \sum_{\substack{n=1 \\
n \neq u, l}} \sum_{s \in S_{n}} \rho_{v s}^{(u),(n)} \rho_{s q}^{(n),(l)}\right)\right], \\
& N_{3}=w_{q,(2)}^{(l)} \sum_{\substack{u=1 \\
u \neq l}}^{K} \sum_{v \in S_{u}} \rho_{q v}^{(i)}-w_{k,(3)}^{(i)} \sum_{\substack{l=1 \\
l \neq i}}^{K} \sum_{q \in S_{l}} \rho_{k q}^{(i),(l)}\left(Z_{q}^{(l)} Z_{v}^{(u)}\right) . \\
&(26)
\end{aligned}
$$

After coherent combining using $\overline{H_{k}^{(i)}}$, the final output is given by

$$
\overline{H_{k}^{(i)}} Y_{k,(3)}^{(i)}=\left|H_{k}^{(i)}\right|^{2} X_{k}^{(i)} F+I_{3}^{\prime}+N_{3}^{\prime}
$$

where $I_{3}^{\prime}=\overline{H_{k}^{(i)}} I_{3}$ and $N_{3}^{\prime}=\overline{H_{k}^{(i)}} N_{3}$. Again, assuming $H_{k 1}^{(i)}$ and $H_{k 2}^{(i)}$ to be uncorrelated, we can obtain the variance of $I_{3}^{\prime}$, $\sigma_{I_{3}^{\prime}}^{2}$, and the variance of $N_{3}^{\prime}, \sigma_{N_{3}^{\prime}}^{2}$, respectively, as

$$
\sigma_{I_{3}^{\prime}}^{2}=\left|H_{k}^{(i)}\right|^{2} \sigma_{I_{3}}^{2},
$$

where

$$
\begin{aligned}
\sigma_{I_{3}}^{2}= & \sum_{l=1}^{K} \sum_{\substack{q \in S_{l} \\
q \neq k}} \mid \rho_{k q}^{(i),(l)} \\
& \cdot\left[\left(1-w_{k,(3)}^{(i)}\left(1-w_{q,(2)}^{(l)} \sum_{\substack{u=1 \\
u \neq l}}^{K} \sum_{v \in S_{u}} \rho_{q v}^{(l),(u)} \rho_{v q}^{(u),(l)}\right)\right)\right. \\
& -w_{k,(3)}^{(i)} \sum_{\substack{u=1 \\
u \neq i, l}}^{K} \sum_{v \in S_{u}} \rho_{k v}^{(l),(u)}\left(\rho_{v q}^{(u),(l)}\left(1-w_{v,(2)}^{(u)}\right)\right. \\
& \left.\left.-w_{v,(2)}^{(u)} \sum_{\substack{n=1 \\
n \neq u, l}}^{K} \sum_{s \in S_{n}} \rho_{v s}^{(u),(n)} \rho_{s q}^{(n),(l)}\right)\right]\left.\right|^{2},
\end{aligned}
$$

and

$$
\sigma_{N_{3}^{\prime}}^{2}=\left|H_{k}^{(i)}\right|^{2} \sigma_{N_{3}}^{2}
$$

where $\sigma_{N_{3}}^{2}$ is given by Eqn. (33) (see top of next page). The average SIR on the $k$ th subcarrier of the $i$ th user at the output of the 3nd stage, $S I R_{k,(3)}^{(i)}$, can then be obtained as

$$
S_{I R,(3)}^{(i)}=\frac{F^{2}}{\sigma_{I_{3}^{\prime}}^{2}+\sigma_{N_{3}^{\prime}}^{2}}
$$

\section{SIR Results and Discussions}

In Fig. 3, we plot the average SIR at the output of the 2nd stage as a function of weights $w_{k,(2)}^{(i)}$ obtained through both analysis (Eqn. 23) as well as simulations. The system parameters considered are: $N=32, K=4,\left[\epsilon_{1}, \epsilon_{2}, \epsilon_{3}, \epsilon_{4}\right]=$ $[-0.1,0.3,0.25,-0.15]$, and $\mathrm{SNR}=25 \mathrm{~dB}$. The channel model used is a one sample spaced, two-ray, equal-gain Rayleigh fading model. Perfect knowledge of $\epsilon$ 's is assumed. Average SIR for both block allocation as well as interleaved allocation are plotted. The SIRs in the simulations are measured as follows. For a given realization of the channel coefficients $H_{k}^{(i)}$ 's, $i$ ) the total power in the received signal (i.e., power in the LHS in Eqns. (17) and (28) is computed, ii) using the knowledge of $H_{k}^{(i)}$ and $\rho$ 's, the desired signal power is computed (i.e., power in the 1st term on RHS in Eqns. (17) and (28), $i$ ii) the difference between powers in $i$ ) and $i i$ ) gives the interference plus noise power, and $i v$ ) SIR is computed as the ratio of the powers in $i i$ ) and $i i i$ ). The average SIR is obtained over several realizations of the channel coefficients. The difference between the simulated SIR and the analytical SIR is that in the analysis to derive the interference variance it is assumed that $H_{k 1}^{(i 1)}$ and $H_{k 2}^{(i 2)}, k 1 \in S_{i 1}, k 2 \in S_{i 2}, i 1 \neq i 2$, are uncorrelated, whereas this assumption is not there in the simulations.

The following observations can be made from Fig. 3. First, for the considered channel model and system parameters, block allocation results in a higher output SIR than interleaved 


$$
\begin{aligned}
& \sigma_{N_{3}}^{2}=\sigma^{2}\left[N\left(1+w_{k,(3)}^{(i)} \sum_{\substack{l=1 \\
l \neq i}}^{K} \sum_{q \in S_{l}} \rho_{k q}^{(i),(l)} w_{q,(2)}^{(l)} \rho_{q k}^{(l),(i)}\right)^{2}\right. \\
& +N\left(w_{k,(3)}^{(i)}\right)^{2} \sum_{\substack{u=1 \\
u \neq i}}^{K} \sum_{r \in S_{u}}^{K}\left|\left(-\rho_{k r}^{(j),(u)}+\sum_{\substack{l=1 \\
l \neq i, u}}^{K} \sum_{q \in S_{l}} \rho_{k q}^{(i),(l)} \rho_{q r}^{(l),(i)} w_{q,(2)}^{(l)}\right)\right|^{2}+N\left(w_{k,(3)}^{(i)}\right)^{2} \sum_{r \in S_{i}}\left|\sum_{\substack{l=1 \\
l \neq i}}^{K} \sum_{q \in S_{l}} \rho_{k q}^{(i),(l)} \rho_{q r}^{(l),(i)} w_{q,(2)}^{(l)}\right|^{2} \\
& +\left(w_{k,(3)}^{(i)}\right)^{2} \sum_{\substack{u=1 \\
u \neq i}}^{K} \sum_{r \in S_{u}} \sum_{\substack{c=1 \\
c \neq i, u}}^{K} \sum_{v \in S_{c}}\left(-\rho_{k r}^{(i),(u)}+\sum_{\substack{l=1 \\
l \neq i, u}}^{K} \sum_{q \in S_{l}} \rho_{k q}^{(i),(l)} \rho_{q r}^{(l),(i)} w_{q,(2)}^{(l)}\right) \overline{\left(-\rho_{k v}^{(i),(c)}+\sum_{\substack{l=1 \\
l \neq i, c}}^{K} \sum_{q \in S_{l}} \rho_{k q}^{(i),(l)} \rho_{q v}^{(l),(i)} w_{q,(2)}^{(l)}\right)} \eta_{r v}^{(u),(c)} \\
& +2 w_{k,(3)}^{(i)} \mathcal{R} e\left(\sum_{\substack{u=1 \\
u \neq i}}^{K} \sum_{r \in S_{u}} \sum_{v \in S_{i}}\left(-\rho_{k r}^{(i),(u)}+\sum_{\substack{l=1 \\
l \neq i, u}}^{K} \sum_{q \in S_{l}} \rho_{k q}^{(i),(l)} \rho_{q r}^{(l),(i)} w_{q,(2)}^{(l)}\right) \eta_{r v}^{(u),(i)} \overline{\left.\sum_{\substack{l=1 \\
l \neq i}}^{K} \sum_{q \in S_{l}} \rho_{k q}^{(i),(l)} \rho_{q r}^{(l),(i)} w_{q,(2)}^{(l)}\right)}\right. \\
& \left.+2 w_{k,(3)}^{(i)} \mathcal{R} e\left(\sum_{\substack{u=1 \\
u \neq i}}^{K} \sum_{r \in S_{u}} \eta_{r k}^{(u),(i)}\left(1+w_{k,(3)}^{(i)} \sum_{\substack{l=1 \\
l \neq i}}^{K} \sum_{q \in S_{l}} \rho_{k q}^{(i),(l)} w_{q,(2)}^{(l)} \rho_{q k}^{(l),(i)}\right) \overline{\left.\left(-\rho_{k r}^{(i),(u)}+\sum_{\substack{l=1 \\
l \neq i, u}}^{K} \sum_{q \in S_{l}} \rho_{k q}^{(i),(l)} \rho_{q r}^{(l),(i)} w_{q,(2)}^{(l)}\right)\right)}\right)\right] .
\end{aligned}
$$

allocation. Second, the match between the analytical SIR and simulated SIR is quite good implying that the uncorrelated assumption on $H_{k}^{(i)}$,s is reasonable. Third, the maximum average output SIR occurs at an optimum weight (maximum SIR of about $15 \mathrm{~dB}$ at $w_{k,(2)}^{(i)} \approx 0.7$ for interleaved allocation and a maximum SIR of about $21 \mathrm{~dB}$ at $w_{k,(2)}^{(i)} \approx 0.6$ for block allocation). This implies that the average SIR expressions in (23) and (32) can be maximized to obtain optimum weights. Closed-form expressions for the optimum weights are derived in the Appendix.

\section{RESUlTS AND DisCUSSION}

In this section, we present the numerical and simulation results of the average SIR and BER performance of the proposed WLPIC scheme and compare with those of other detectors in the recent literature. The channel model used throughout this section is a one sample spaced, two-ray, equal-gain Rayleigh fading model. Also, perfect knowledge of CFO values is assumed. In Fig. 4, we plot the analytically computed average SIR as a function of the subcarrier index $k=1,2, \cdots, N$ under no noise condition (i.e., $\sigma^{2}=0$ ) for a) SUD, $b$ ) 2nd and 3rd stages of the CLPIC scheme (where $\left.w_{k,(2)}^{(i)}=w_{k,(3)}^{(i)}=1, \forall i, k\right)$, and $\left.c\right)$ 2nd and 3rd stages of the WLPIC scheme, in an uplink OFDMA system with $N=32$ subcarriers, $K=4$ users, interleaved allocation, and CFOs of the different users $\left[\epsilon_{1}, \epsilon_{2}, \epsilon_{3}, \epsilon_{4}\right]=[-0.1,0.3,0.25,-0.15]$.

From Fig. 4, it can be seen that the SUD gives the least SIRs in all subcarriers since no interference cancellation is performed. When interference cancellation is performed using CLPIC scheme (where unity weights are used), the 2nd stage output SIR improves significantly compared to that of SUD. The CLPIC 3rd stage output SIR improves further compared to the CLPIC 2nd stage output SIR. The WLPIC scheme (where the optimized weights derived in the Appendix are used) performs significantly better than both SUD as well as CLPIC. For example, the 3rd stage of the WLPIC results in an average SIR of about $23 \mathrm{~dB}$ on all the subcarriers which is

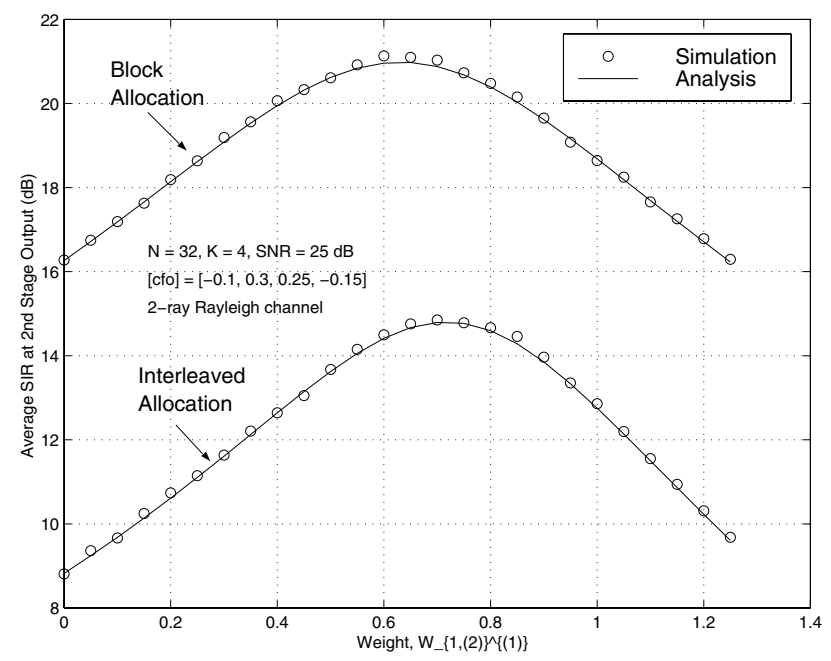

Fig. 3. Average SIR of the 1st user at the output of the 2nd stage of the proposed WLPIC scheme as a function of the weight on the 1st subcarrier, $w_{1,(2)}^{(1)}$. $N=32, K=4, \mathrm{SNR}=25 \mathrm{~dB},\left[\epsilon_{1}, \epsilon_{2}, \epsilon_{3}, \epsilon_{4}\right]=[-0.1,0.3,0.25,-0.15]$. Interleaved and block allocation. Analysis vs simulation.

significantly larger than those in the other detectors. Thus the performance benefit of using the optimized weights in WLPIC instead of unity weights as in CLPIC or zero weights as in SUD is clearly evident in Fig. 4.

For the same set of parameters in Fig. 4, we plot the simulated BER performance of SUD, CLPIC (2nd and 3rd stages) and WLPIC (2nd and 3rd stages) in Fig. 5 for BPSK. The single user performance (no MUI) is also shown for comparison purposes. From Fig. 5, it can be seen that the proposed WLPIC scheme results in significantly better BER performance than both the SUD as well as the CLPIC scheme. The 3rd stage of the WLPIC scheme is found to approach the single user (no MUI) performance. We have observed similar SIR and BER improvement for the case of block allocation as well as 16-QAM.

We note that the $\epsilon$ values we have used in the above example 


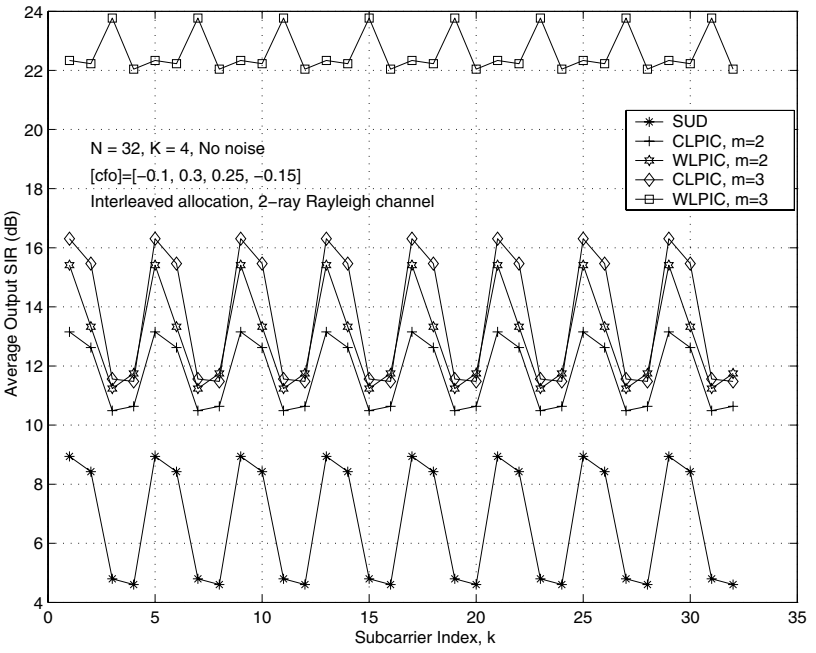

Fig. 4. Average SIR as a function of subcarrier index, $k$, for different detectors. $N=32, K=4,\left[\epsilon_{1}, \epsilon_{2}, \epsilon_{3}, \epsilon_{4}\right]=[-0.1,0.3,0.25,-0.15]$. No noise $\left(\sigma^{2}=0\right)$. Interleaved allocation. Analysis.

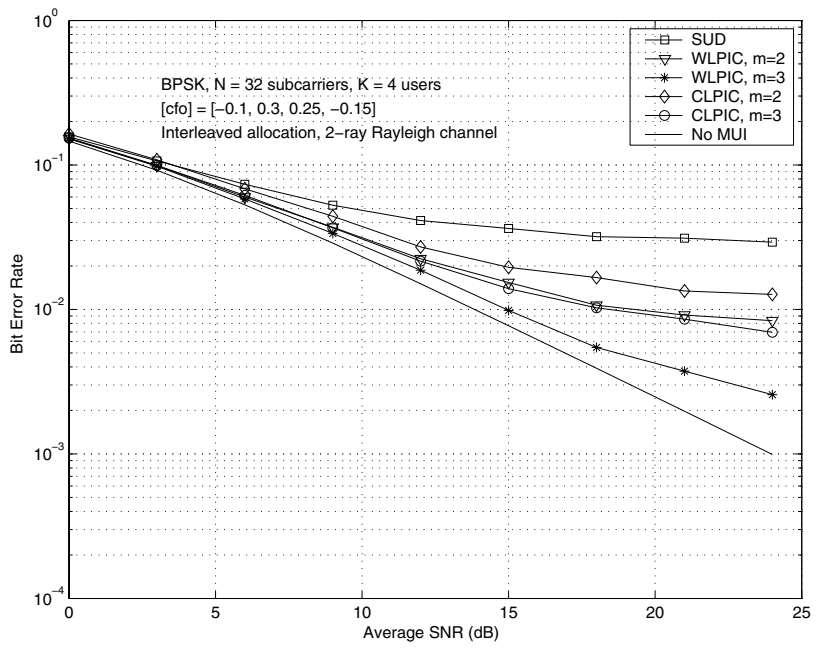

Fig. 5. Bit error rate performance of the proposed WLPIC scheme for BPSK. $N=32, K=4,\left[\epsilon_{1}, \epsilon_{2}, \epsilon_{3}, \epsilon_{4}\right]=[-0.1,0.3,0.25,-0.15]$. Interleaved allocation. Simulation.

(and also in subsequent examples in this section) are high (e.g., $|\epsilon|>0.1$ ) compared to the CFO limits specified in current OFDMA-based standards. For example, the IEEE 802.16e standard [19], which defines a 2048 subcarrier uplink OFDMA system with a subcarrier spacing of $9.8 \mathrm{KHz}$, specifies that the transmit carrier frequency at the user be synchronized to the BS with a maximum tolerance of $1 \%$ of the subcarrier spacing (i.e., $\epsilon$ must be $<0.01$ ), which is achieved using long preambles and closed-loop frequency correction between user transmitter and BS receiver. For such small CFO values $(\epsilon<$ 0.01 ), the resulting MUI and the associated performance loss in using an SUD is small. The advantage of using interference cancellers at the BS, however, is that larger $\mathrm{CFO}$ values can be tolerated at the BS receiver, which in turn can allow the use of low-cost, free-running transmit oscillators at the users that can result in reduction of user terminal cost.

\section{A. Comparison With HLCC and CLJL Schemes}

In this subsection, we present a comparison of the performance and complexity of the proposed WLPIC scheme with other detectors reported in the recent literature, namely, $a$ ) the HLCC scheme in [9], b) CLJL scheme in [6], and c) SUD. It is noted that while the proposed WLPIC and the HLCC schemes are essentially interference cancellers, the CLJL and SUD schemes are detectors without interference cancellation.

1) SIR and BER Comparison: In the proposed WLPIC scheme, CFO compensation is done in time-domain. Hence, as per Eqns. (6),(7),(8), the performance of the WLPIC scheme is affected by the magnitudes of the differences between the desired user CFO $\left(\epsilon_{i}\right)$ and other user CFOs $\left(\epsilon_{l}\right.$ 's, $\left.l \neq i\right)$, i.e., $\left|\delta_{l i}\right|=\left|\epsilon_{l}-\epsilon_{i}\right|, l, i=1,2, \cdots, K, l \neq i$. On the other hand, since the CFO compensation is done in frequencydomain in the HLCC scheme, as per Eqns. (10), (15) and (22) in [9], the performance of the HLCC scheme is affected by the magnitudes of the individual CFO values of other users, $\left|\epsilon_{l}\right|$ 's, $l=1,2, \cdots, K, l \neq i$. For the same reason of timedomain versus frequency-domain CFO compensation, SUD performance is affected by $\left|\delta_{l i}\right|$ 's whereas CLJL performance is affected by $\left|\epsilon_{l}\right|$ 's. The above observations are illustrated in Figs. 6 and 7. In Fig. 6, we plot the average SIR at the output of SUD and CLJL in a 2-user system $(K=2)$ as a function of $\delta_{21}=\epsilon_{2}-\epsilon_{1}$, with $N=64$, SNR $=10 \mathrm{~dB}$, and interleaved allocation of subcarriers. User 1 is taken as the desired user and user 2 as the interfering user. The following observations can be made from Fig. 6. First, in the case of SUD, $i$ ) the other user CFO, i.e., $\epsilon_{2}$, affects the SUD performance only through $\delta_{21}$, regardless of the individual value of $\epsilon_{2}, i i$ ) MUI is perfectly cancelled when $\epsilon_{2}=\epsilon_{1}$ even if these $\epsilon$ values are individually large (i.e., output $\mathrm{SIR}=\mathrm{SNR}=10 \mathrm{~dB}$ for $\delta_{21}=0$ ), and $\left.i i i\right)$ the output SIR degrades as $\left|\delta_{21}\right|$ increases (e.g., SIR degradation is about $1 \mathrm{~dB}$ and $3 \mathrm{~dB}$ for $\left|\delta_{21}\right|$ of 0.1 and 0.2 , respectively). Second, in the case of CLJL, $i$ ) the output SIR depends on the individual value of $\epsilon_{2}$ in addition to the value of $\left.\delta_{21}, i i\right)$ SIR degrades as $\left|\epsilon_{2}\right|$ increases (e.g., observe that $\left|\epsilon_{2}\right|=0.05$ results in larger output SIRs compared to $\left|\epsilon_{2}\right|=0.15$, and $i i i$ ) the best SIR occurs when $\epsilon_{1}=0$ (e.g., for $\epsilon_{2}=0.05$, the maximum SIR of about $9.7 \mathrm{~dB}$ occurs at $\left.\delta_{21}=0.05\right)$. Third, cross-overs between the performance of SUD and CLJL occur depending on $\left|\delta_{21}\right|$ and $\left|\epsilon_{2}\right|$. For example, SUD performs better than CLJL when $\left|\delta_{21}\right|<0.05$ and $\left|\epsilon_{2}\right|=0.05$, as well as when $\left|\delta_{21}\right|<0.15$ and $\left|\epsilon_{2}\right|=0.15$. That is, SUD performs better when $\left|\delta_{21}\right|<\left|\epsilon_{2}\right|$ and CLJL performs better when $\left|\epsilon_{2}\right|<\left|\delta_{21}\right|$. A similar cross-over in performance between the 2nd stages of WLPIC and HLCC schemes is also observed in Fig. 7. Further, comparing Figs. 6 and 7 it can be observed that, because of the interference cancellation they perform, WLPIC and HLCC schemes result in larger output SIRs compared to SUD and CLJL schemes.

In Figs. 8 and 9, we present a comparison of the SIR performance of various detectors for a $K=4$ user system with $N=64$, interleaved allocation, and no noise. We consider two cases of $\mathrm{CFO}$ values, namely, CFO-1 $=\left[\epsilon_{1}, \epsilon_{2}, \epsilon_{3}, \epsilon_{4}\right]=[0.1,-0.1,-0.05,0.05]$ and CFO-2 = $\left[\epsilon_{1}, \epsilon_{2}, \epsilon_{3}, \epsilon_{4}\right]=[0.15,0.12,0.16,0.08]$. We note that the CFO-1 values in the above are the same ones used in the 


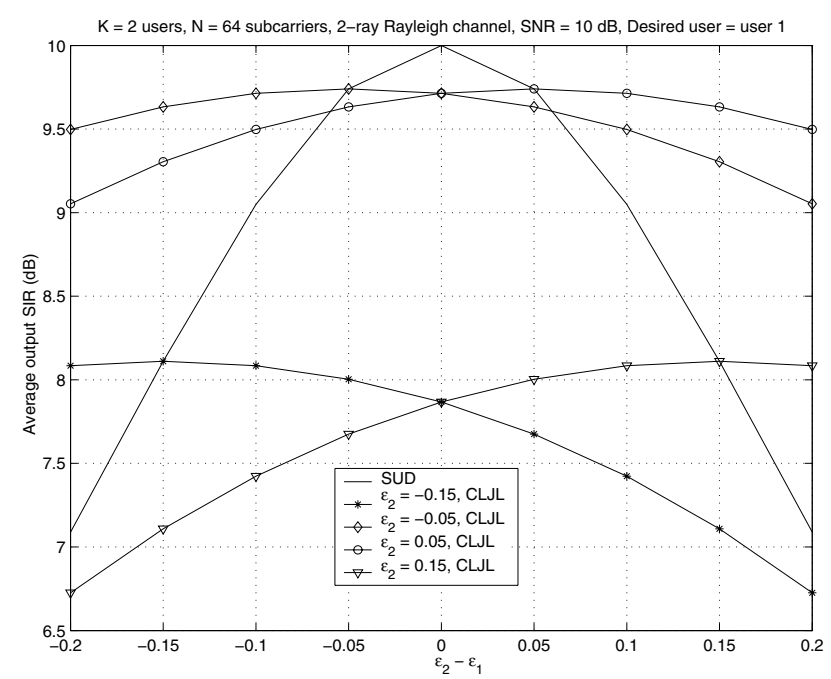

Fig. 6. Average output SIR versus $\delta_{21}=\epsilon_{2}-\epsilon_{1}$ performance comparison between SUD and CLJL. $K=2, N=64$, SNR $=10 \mathrm{~dB}$. Interleaved allocation.

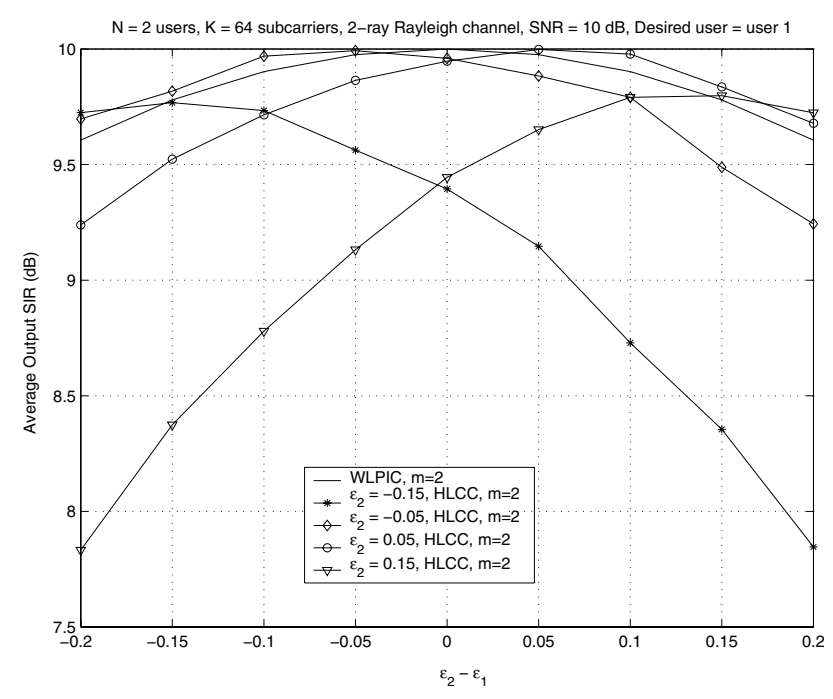

Fig. 7. Average output SIR versus $\delta_{21}=\epsilon_{2}-\epsilon_{1}$ performance comparison between the 2 nd stages of WLPIC and HLCC schemes. $K=2, N=64$, $\mathrm{SNR}=10 \mathrm{~dB}$. Interleaved allocation.

performance plots of [9]. Fig. 8 is for CFO-1 and Fig. 9 is for CFO-2. It can be seen that HLCC performs better than WLPIC in the case of CFO-1 (see Fig. 8), whereas WLPIC performs better than HLCC in the case of CFO-2 (see Fig. 9). This can be attributed to the fact that in the case of CFO-1, the magnitudes of CFO differences are large compared to the magnitude of individual CFOs. For a desired user $i$, this can be seen by comparing the sum of CFO differences, $\Lambda_{\delta}^{(i)}$, given by

$$
\Lambda_{\delta}^{(i)} \triangleq \sum_{l=1, l \neq i}^{K}\left|\delta_{l i}\right|,
$$

and the sum of individual CFOs, $\Lambda_{\epsilon}^{(i)}$, given by

$$
\Lambda_{\epsilon}^{(i)} \triangleq \sum_{l=1, l \neq i}^{K}\left|\epsilon_{l}\right| .
$$

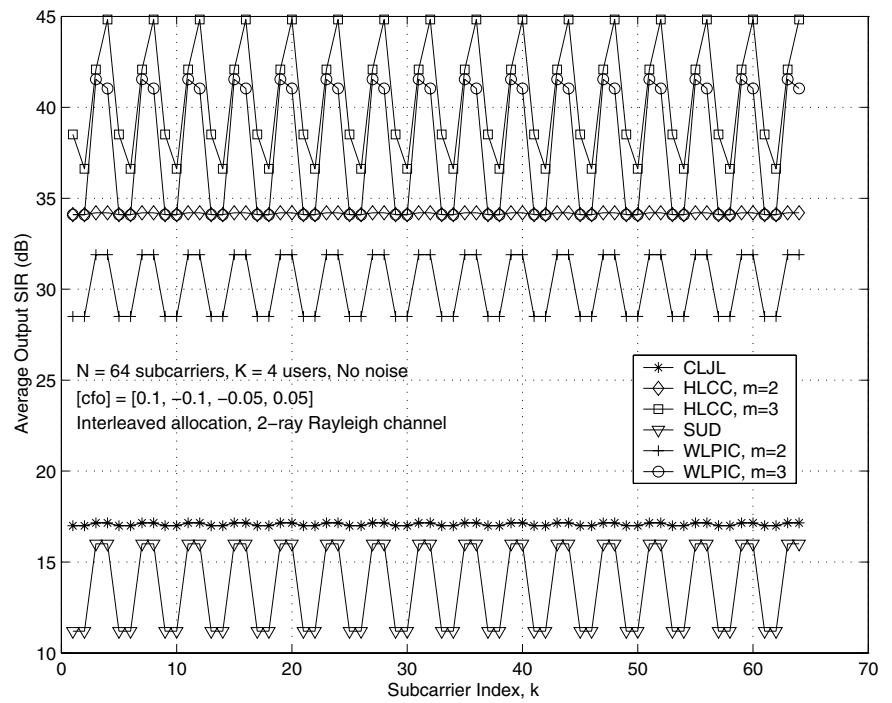

Fig. 8. Comparison of the SIR performance of the proposed WLPIC scheme with HLCC and CLJL schemes. $N=64, K=4$, CFO-1 $=[\mathbf{0 . 1},-\mathbf{0 . 1},-\mathbf{0 . 0 5}, \mathbf{0 . 0 5}]$. No noise $\left(\sigma^{2}=0\right)$. Interleaved allocation. Analysis. HLCC performs better than WLPIC.

For CFO-1, $\Lambda_{\delta, c f o 1}^{(1)}=\sum_{l=2}^{4}\left|\delta_{l 1}\right|=0.4$ and $\Lambda_{\epsilon, c f o 1}^{(1)}=$ $\sum_{l=2}^{4}\left|\epsilon_{l}\right|=0.2$. Since $\Lambda_{\epsilon, c f o 1}^{(1)}<\Lambda_{\delta, c f o 1}^{(1)}$, individual CFOs are small and hence HLCC performs better. For CFO-2, $\Lambda_{\delta, c f o 2}^{(1)}=0.11$ and $\Lambda_{\epsilon, c f o 2}^{(1)}=0.36$, and WLPIC performs better in this case since $\Lambda_{\delta, c f o 2}^{(1)}<\Lambda_{\epsilon, c f o 2}^{(1)}$. In terms of BER performance also, we have observed that HLCC performs better in CFO-1 whereas WLPIC performs better in CFO-2.

In practice, the $\mathrm{CFO}$ values at the receiver can be arbitrary. In order to make the receiver robust under various CFO conditions, for a desired user $i$, the receiver operation can be switched between WLPIC and HLCC depending on the computed values of $\Lambda_{\delta}^{(i)}$ and $\Lambda_{\epsilon}^{(i)}$ as follows:

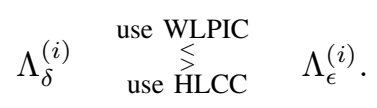

The above mechanism can enable the receiver to achieve the better performance among WLPIC and HLCC schemes under various $\mathrm{CFO}$ conditions. An example illustrating this is given in Figs. 10 and 11, where the BER performance of 16-QAM for a $K=8$ user system with 64 subcarriers and interleaved allocation are plotted. In Fig. 10, the CFO values of the different users are taken to be CFO-3 $=[-0.05,0.08,0.11,-0.04,0.07,-0.06,0.12,0.05]$. In Fig. 11, the CFO values considered are CFO-4 = $[0.10,0.15,0.07,0.12,0.14,-0.03,-0.01,0.16]$. In CFO-3, $\left(\Lambda_{\delta, c f o 3}^{(1)}=0.7\right)>\left(\Lambda_{\epsilon, c f o 3}^{(1)}=0.53\right)$, and hence as per the selection rule in (36), HLCC operation is chosen which results in better BER performance than WLPIC as seen in Fig. 10. In CFO-4, on the other hand, $\left(\Lambda_{\delta, c f o 4}^{(1)}=0.44\right)<\left(\Lambda_{\epsilon, c f o 4}^{(1)}=\right.$ $0.68)$, and hence WLPIC operation is chosen which performs better than HLCC as seen in Fig. 11.

We also carried out a comparison study of the various detectors in terms of coded frame error rate (FER) performance. We considered a rate-1/2 convolutional code with constraint length 5 . The system parameters considered include $K=4$ users, $N=64$ subcarriers, CFO-5 $=[\mathbf{0 . 2 2}, \mathbf{0 . 2}, \mathbf{0 . 1 8}, \mathbf{0 . 1 5}]$, 


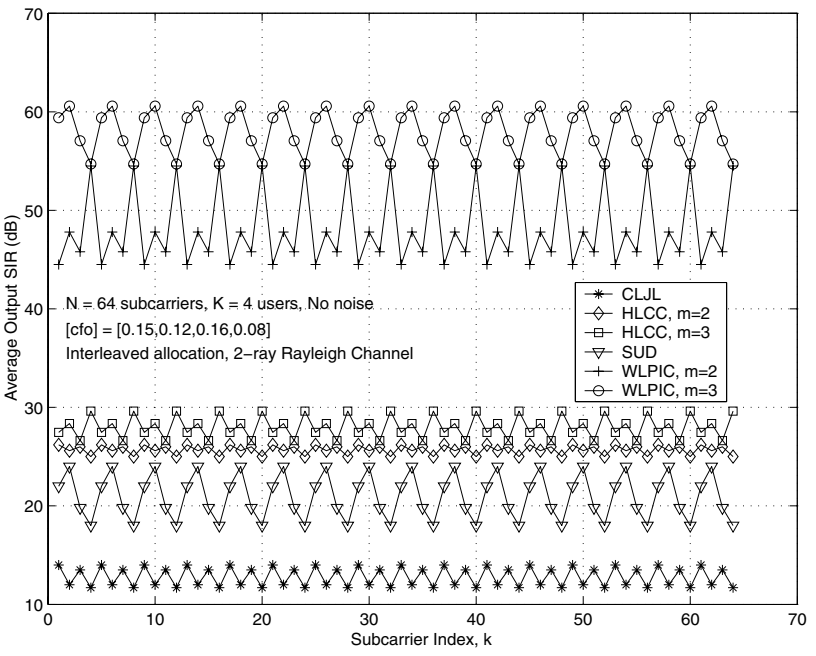

Fig. 9. Comparison of the SIR performance of the proposed WLPIC scheme with HLCC and CLJL schemes. $N=64, K=4$, CFO-2 $=[\mathbf{0 . 1 5}, \mathbf{0 . 1 2}, \mathbf{0 . 1 6}, \mathbf{0 . 0 8}]$. No noise $\left(\sigma^{2}=0\right)$. Interleaved allocation. Analysis. WLPIC performs better than HLCC.

TABLE I

COMPLEXITY COMPARISON AMONG DIFFERENT DETECTORS. $N$ : NUMBER OF SUBCARRIERS IN THE SYSTEM, $K$ : NUMBER OF USERS IN THE SYSTEM, $m$ : STAGE INDEX.

\begin{tabular}{|l|l|}
\hline Detector & Complexity (\# complex multiplications) \\
\hline \hline CLJL scheme & $\frac{N}{2} \log N+\frac{N^{2}}{K}$ \\
\hline HLCC scheme & $\frac{N}{2} \log N+\frac{N^{2}}{K}+(m-1)\left[N^{2}+\frac{N^{2}}{K}\right]$ \\
\hline SUD scheme & $\frac{K N}{2} \log N-\left[\frac{K N}{2} \log K-\frac{3}{2}(K-1) N\right]$ \\
\hline WLPIC scheme & $\frac{K N}{2} \log N-\left[\frac{K N}{2} \log K-\frac{3}{2}(K-1) N\right]$ \\
& $+(m-1)\left[N^{2}-\frac{N^{2}}{K}\right]$ \\
\hline
\end{tabular}

interleaved allocation, 4-QAM, and 2-ray Rayleigh fading channel. As in [9], each frame consists of 10 OFDM symbols, and it is assumed that the channels do not vary within one frame but vary from frame to frame. In each frame, an $8 \times 40$ block bit interleaver is employed. Fig. 12 shows the simulated coded FER performance for various detectors. For this system scenario, $\left(\Lambda_{\delta, c f o 5}^{(1)}=0.13\right)<\left(\Lambda_{\epsilon, c f o 5}^{(1)}=0.53\right)$, and hence WLPIC scheme performs better than HLCC scheme. Likewise, SUD performs better than CLJL scheme in this scenario. As in [9], we carried out simulations with imperfect CFO estimates. While imperfect CFO estimates degraded the performance as in [9], we observed similar comparative performance behavior between WLPIC and HLCC as in the case of perfect CFO estimates in the above.

2) Complexity Comparison: In addition to the above SIR and BER/FER performance comparison, we carried out a complexity comparison among the different detectors. The complexities of various detectors in terms of number of complex multiplications required are listed in Table I. The complexities of CLJL and SUD schemes are same as those given in [6]. Compared to the CLJL scheme, HLCC scheme has an additional complexity of $N^{2}+N^{2} / K$ per cancellation stage (as per Eqns. (18), (19) in [9]). Likewise, compared to the SUD scheme, WLPIC scheme has an additional complexity of $N^{2}-N^{2} / K$ per cancellation stage (as per Eqn. (12)). The complexity comparison between HLCC and WLPIC schemes as a function of number of subcarriers, $N$, for $K=16$ users and $m=2,3$ (2nd, 3rd stages) is shown in Fig. 13. It can be seen that for a given $K$, HLCC scheme is less complex for small $N$, whereas WLPIC scheme has lesser complexity than HLCC scheme for large $N$ (which is typical in OFDMA systems). For example, for $N=1024, K=16$ and $m=2$, HLCC has a complexity of $11,84,768$, whereas WLPIC has a lesser complexity of $10,55,232$. It is further noted that complexity reduction techniques similar to those given in [9] for HLCC scheme (e.g., by way of ignoring weak subcarriers or other user subcarriers far-off from desired user's subcarriers) can be done for the WLPIC scheme as well.

\section{CONCLUSION}

We presented the design and analysis of an interference cancellation scheme for MUI mitigation in uplink OFDMA. The proposed scheme performed CFO compensation in timedomain, followed by DFT operations (on a per-user basis) and multistage linear parallel interference cancellation on these DFT outputs. Estimates of the MUI for cancellation were obtained using soft values of the outputs from the previous stages. We scaled the MUI estimate by weights before cancellation. We derived expressions for the average SIR at the output of the 2nd and 3rd stages of the proposed scheme. While these SIR expressions quantified the improvement in output SIR from one stage to the next, they were also used to obtain the optimum weights in-closed form. The proposed scheme was shown to effectively cancel the MUI caused by the other user CFOs. We showed that the scheme proposed by Huang and Letaief (HLCC scheme) performs better than our scheme when the individual CFO values are small, whereas our scheme performs better than the HLCC scheme when the CFO differences are small (even if the individual CFO values are large). Also, our scheme has lesser complexity than HLCC scheme when the number of subcarriers is large (which is typical in OFDMA systems). Simple metrics based on the knowledge of CFO were proposed to choose between WLPIC and HLCC operation at the receiver so that better performance among these two approaches is achieved under various CFO conditions.

\section{APPENDIX}

\section{OPTIMUM WEIGHTS IN CLOSED-FORM}

The average SIR expressions for 2nd and 3rd stage outputs in (23) and (32) can be maximized to obtain optimum weights for scaling the interference estimate at the 2 nd and 3rd stages.

\section{A. $w_{k,(2)}^{(i), o p t}$ in Closed-Form}

An expression for the optimum weights $w_{k,(2)}^{(i), o p t}$ can be obtained by differentiating (23) w.r.t. $w_{k,(2)}^{(i)}$ and equating to 


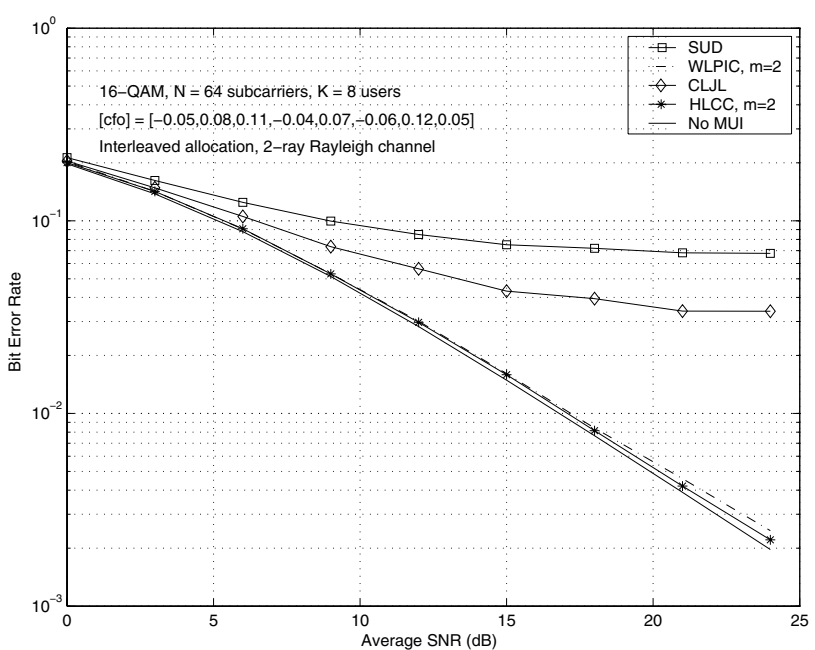

Fig. 10. Bit error rate as a function of average SNR for different detectors for 16-QAM. $K=8, N=64, \mathbf{C F O}-3$ $=[-0.05,0.08,0.11,-0.04,0.07,-0.06,0.12,0.05]$. Interleaved allocation. Simulation. HLCC performs better than WLPIC.

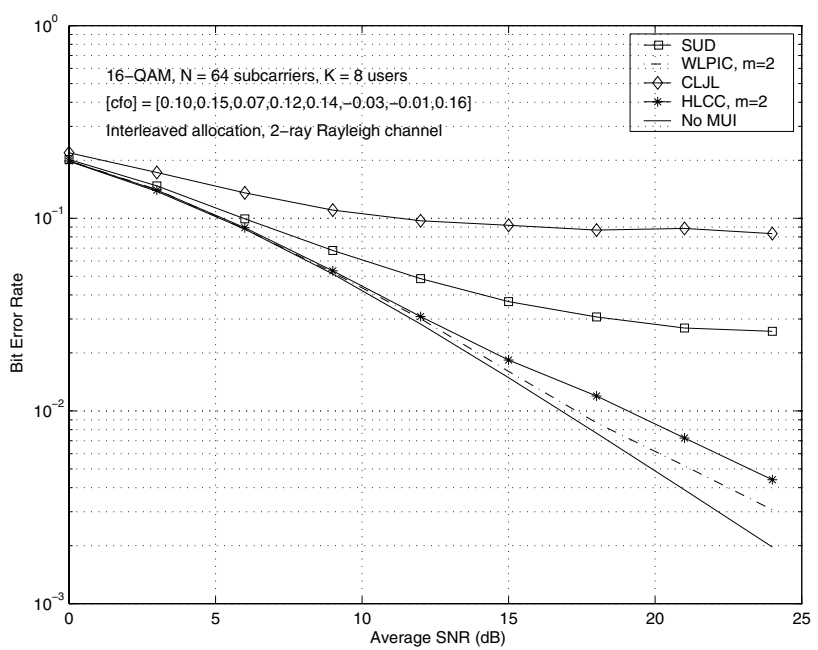

Fig. 11. Bit error rate as a function of average SNR for different detectors for 16-QAM. $K=8, N=32$, CFO-4 $=[0.10,0.15,0.07,0.12,0.14,-0.03,-0.01,0.16]$. Interleaved allocation. Simulation. WLPIC performs better than HLCC.

zero. Accordingly, we obtain the expression for $w_{k,(2)}^{(i),(o p t)}$ as

$$
w_{k,(2)}^{(i), o p t}=-\frac{\left(\beta_{1}+2 \beta_{2} \beta_{3}\right)}{\left(\beta_{1} \beta_{2}+2 \beta_{4}\right)}
$$

where

$$
\begin{gathered}
\beta_{1}=\alpha_{1}-\sigma^{2}\left(\alpha_{6}+\alpha_{7}\right), \quad \beta_{2}=\sum_{\substack{l=1 \\
l \neq i}}^{K} \sum_{q \in S_{l}} \rho_{k q}^{(i),(l)} \rho_{q k}^{(l),(i)}, \\
\beta_{3}=\alpha_{2}+\sigma^{2} N, \quad \beta_{4}=\alpha_{3}+\sigma^{2}\left(N \alpha_{4}+\alpha_{5}\right), \\
\alpha_{1}=2 \sum_{u=1}^{K} \sum_{\substack{r \in S_{u} \\
r \neq k}}\left|\rho_{k r}^{(i),(u)}\right|^{2} \\
+2 \mathcal{R} e\left(\sum_{\substack { u=1 \\
\begin{subarray}{c}{r \in S_{u} \\
r \neq k{ u = 1 \\
\begin{subarray} { c } { r \in S _ { u } \\
r \neq k } }\end{subarray}}^{K} \sum_{\substack{l=1 \\
l \neq i, u}}^{K} \sum_{q \in S_{l}} \rho_{k q}^{(i),(l)} \rho_{q r}^{(l),(u)}\right),
\end{gathered}
$$

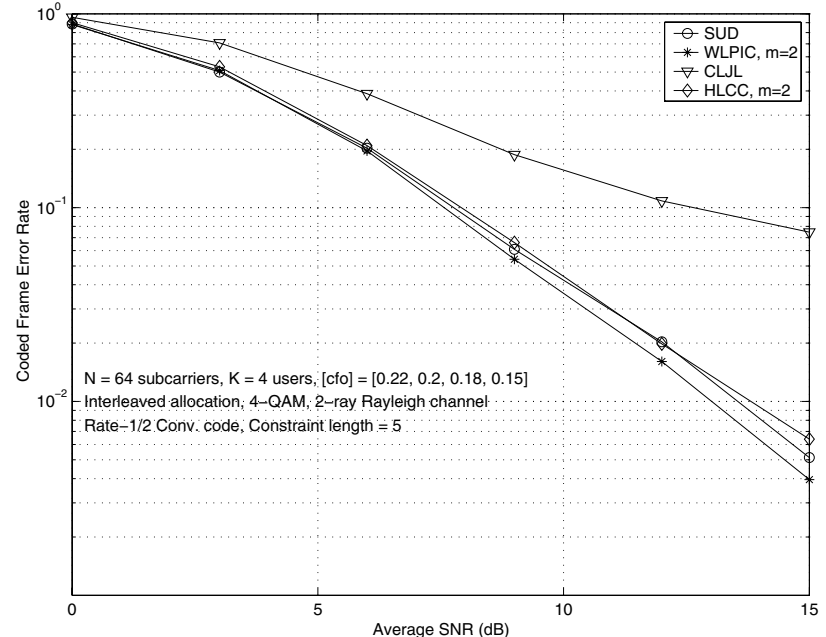

Fig. 12. Coded FER performance comparison among different detectors. $K=4, N=64, \mathbf{C F O}-\mathbf{5}=[\mathbf{0 . 2 2}, \mathbf{0 . 2}, \mathbf{0 . 1 8}, \mathbf{0 . 1 5}]$. Interleaved allocation, 4-QAM, rate-1/2 convolutional code, constraint length $=5$. Simulation. WLPIC performs better than HLCC.

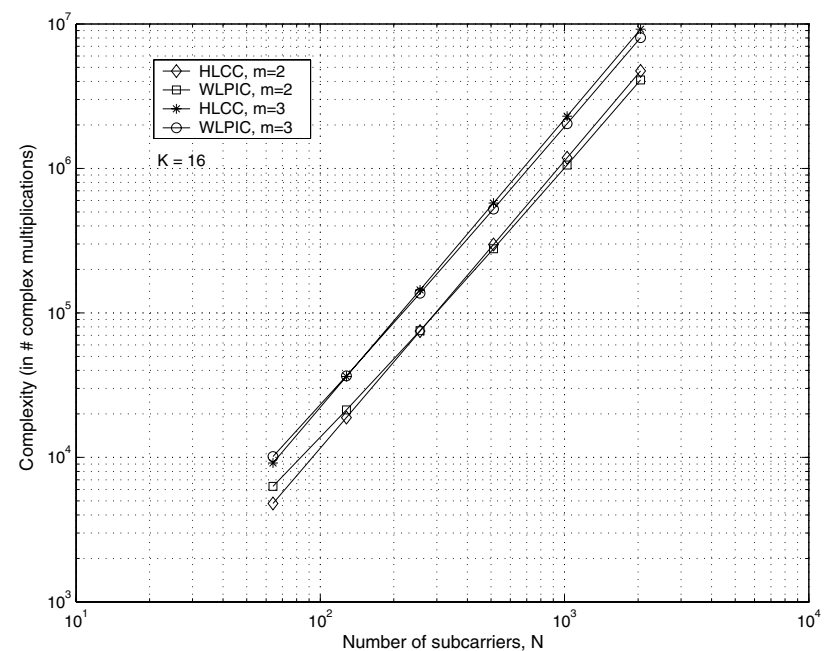

Fig. 13. Complexity comparison of the proposed WLPIC scheme with HLCC scheme. $K=16, m=2,3$.

$$
\alpha_{2}=\sum_{u=1}^{K} \sum_{\substack{r \in S u \\ r \neq k}} \mid\left(\left.\rho_{k r}^{(i),(u)}\right|^{2}\right.
$$

$$
\alpha_{3}=\sum_{u=1}^{K} \sum_{\substack{r \in S u \\ r \neq k}}\left|\left(\rho_{k r}^{(i),(u)}+\sum_{\substack{l=1 \\ l \neq i, u}}^{K} \sum_{q \in S_{l}} \rho_{k q}^{(i),(l)} \rho_{q r}^{(l),(u)}\right)\right|^{2},
$$$$
\alpha_{4}=\sum_{\substack{l=1 \\ l \neq i}}^{K} \sum_{q \in S_{l}}\left|\rho_{k q}^{(i),(l)}\right|^{2}
$$

$$
\begin{gathered}
\alpha_{5}=\sum_{\substack{l=1 \\
l \neq i}}^{K} \sum_{q \in S_{l}} \sum_{\substack{c=1 \\
c \neq i, l}}^{K} \sum_{v \in S_{c}} \rho_{k q}^{(i),(l)} \overline{\rho_{k v}^{(i),(c)}} \eta_{q v}^{(l),(c)}, \\
\alpha_{6}=\mathcal{R} e\left(\sum_{\substack{l=1 \\
l \neq i}}^{K} \sum_{q \in S_{l}} \rho_{k q}^{(i),(l)} \eta_{q k}^{(l),(i)}\right),
\end{gathered}
$$




$$
\alpha_{7}=\mathcal{R} e\left(\sum_{\substack{l=1 \\ l \neq i}}^{K} \sum_{q \in S_{l}} \rho_{k q}^{(i),(l)} \eta_{k q}^{(i),(l)}\right)
$$

\section{B. $w_{k,(3)}^{(i), o p t}$ in Closed-form}

Similarly, by differentiating (32) w.r.t. $w_{k,(3)}^{(i)}$ and equating to zero, we obtain the expression for the optimum weights $w_{k,(3)}^{(i), o p t}$, in closed-form, as

$$
w_{k,(3)}^{(i), o p t}=-\frac{\left(\gamma_{1}+2 \gamma_{2} \gamma_{3}\right)}{\left(\gamma_{1} \gamma_{2}+2 \gamma_{4}\right)}
$$

where

$$
\begin{aligned}
& \gamma_{1}=2 \psi_{2 b}+\sigma^{2}\left(2 N \psi_{4 a}+2 \psi_{4 f}+2 \psi_{4 g}\right) \\
& \gamma_{2}=\sum_{\substack{l=1, l \neq i}}^{K} \sum_{q \in S_{l}} \rho_{k q}^{(i),(l)}\left(\rho_{q k}^{(l),(i)}\left(1-w_{q,(2)}^{(l)}\right)\right. \\
& \left.-w_{q,(2)}^{(l)} \sum_{\substack{n=1 \\
n \neq l, i}}^{K} \sum_{s \in S_{n}} \rho_{q s}^{(l),(n)} \rho_{s k}^{(n),(l)}\right) \\
& \gamma_{3}=\psi_{2 c}+N \sigma^{2} \\
& \gamma_{4}=\psi_{2 a}+\sigma^{2}\left(N \psi_{4 a}^{2}+N \psi_{4 b}+\psi_{4 c}+N \psi_{4 d}+2 \psi_{4 a} \psi_{4 f}\right) \\
& \psi_{2 a}=\sum_{u=1}^{K} \sum_{\substack{r \in S_{u} \\
r \neq k}} \mid \rho_{k r}^{(i),(u)}\left(1-w_{r,(2)}^{(u)} \sum_{\substack{n=1 \\
n \neq u}}^{K} \sum_{s \in S_{n}} \rho_{r s}^{(u),(n)} \rho_{s r}^{(n),(u)}\right) \\
& +\sum_{\substack{l=1 \\
l \neq i, u}}^{K} \sum_{q \in S_{l}} \rho_{k l}^{(i),(u)}\left(\rho_{q r}^{(l),(u)}\left(1-w_{q,(2)}^{(l)}\right) w_{q,(2)}^{(l)}\right. \\
& \left.\sum_{\substack{n=1 \\
n \neq l, u}}^{K} \sum_{s \in S_{n}} \rho_{q s}^{(l),(n)} \rho_{s r}^{(n),(u)}\right)\left.\right|^{2} \\
& \psi_{2 b}=\sum_{u=1}^{K} \sum_{\substack{r \in S_{u} \\
r \neq k}}\left(-\left|\rho_{k r}^{(j),(u)}\right|^{2}\right. \\
& \cdot\left(1-w_{r,(2)}^{(u)} \sum_{\substack{n=1 \\
n \neq u}}^{K} \sum_{s \in S_{n}} \rho_{r s}^{(u),(n)} \rho_{s r}^{(n),(u)}\right) \\
& -\mathcal{R} e\left[\overline { \rho _ { k r } ^ { ( i ) , ( u ) } } \sum _ { \substack { l = 1 \\
l \neq i , u } } ^ { K } \sum _ { q \in S _ { l } } \rho _ { k q } ^ { ( l ) , ( u ) } \left(\rho_{q r}^{(l),(u)}\left(1-w_{q,(2)}^{(l)}\right)\right.\right. \\
& \left.\left.\left.-w_{q,(2)}^{(l)} \sum_{\substack{n=1 \\
n \neq l, u}}^{K} \sum_{s \in S_{n}} \rho_{q s}^{(l),(n)} \rho_{s r}^{(n),(u)}\right)\right]\right), \\
& \psi_{2 c}=\sum_{u=1}^{K} \sum_{\substack{r \in S_{u} \\
r \neq k}}\left|\rho_{k r}^{(i),(u)}\right|^{2} \\
& \psi_{4 a}=\sum_{\substack{l=1 \\
l \neq i}}^{K} \sum_{q \in S_{l}} \rho_{k q}^{(i),(l)} w_{q,(2)}^{(l)} \rho_{q k}^{(l),(i)},
\end{aligned}
$$

$$
\begin{aligned}
\psi_{4 b}= & \sum_{\substack{u=1 \\
u \neq i}}^{K} \sum_{r \in S_{u}}^{K} \mid\left(-\rho_{k r}^{(i),(u)}\right. \\
& \left.+\sum_{\substack{l=1 \\
l \neq i, u}}^{K} \sum_{q \in S_{l}} \rho_{k q}^{(i),(l)} \rho_{q r}^{(l),(i)} w_{q,(2)}^{(l)}\right)\left.\right|^{2},
\end{aligned}
$$

$$
\begin{aligned}
& \psi_{4 c}=\sum_{\substack{u=1 \\
u \neq i}}^{K} \sum_{r \in S_{u}} \sum_{\substack{c=1 \\
c \neq i, u}}^{K} \sum_{v \in S_{c}}\left(-\rho_{k r}^{(i),(u)}\right. \\
& \left.+\sum_{\substack{l=1 \\
l \neq i, u}}^{K} \sum_{q \in S_{l}} \rho_{k q}^{(i),(l)} \rho_{q r}^{(l),(i)} w_{q,(2)}^{(l)}\right) \\
& \overline{\left(-\rho_{k v}^{(i),(c)}+\sum_{\substack{l=1 \\
l \neq i, c}}^{K} \sum_{q \in S_{l}} \rho_{k q}^{(i),(l)} \rho_{q v}^{(l),(i)} w_{q,(2)}^{(l)}\right)} \eta_{r v}^{(m),(c)}, \\
& \psi_{4 d}=\sum_{r \in S_{i}}\left|\sum_{\substack{l=1 \\
l \neq i}}^{K} \sum_{q \in S_{l}} \rho_{k q}^{(i),(l)} \rho_{q r}^{(l),(i)} w_{q,(2)}^{(l)}\right|^{2}
\end{aligned}
$$$$
\psi_{4 f}=\mathcal{R} e\left[\sum_{\substack{u=1 \\ u \neq i}}^{K} \sum_{r \in S_{u}} \eta_{r v}^{(u),(i)}\right.
$$$$
\left.\overline{\left(-\rho_{k r}^{(i),(u)}+\sum_{\substack{l=1 \\ l \neq i, u}}^{K} \sum_{q \in S_{l}} \rho_{k q}^{(i),(l)} \rho_{q r}^{(l),(i)} w_{q,(2)}^{(l)}\right)}\right],
$$$$
\psi_{4 g}=\mathcal{R} e\left[\sum _ { \substack { u = 1 \\ u \neq i } } ^ { K } \sum _ { r \in S _ { u } } \sum _ { v \in S _ { i } } \left(-\rho_{k r}^{(i),(u)}\right.\right.
$$$$
\left.+\sum_{\substack{l=1 \\ l \neq i, u}}^{K} \sum_{q \in S_{l}} \rho_{k q}^{(i),(l)} \rho_{q r}^{(l),(i)} w_{q,(2)}^{(l)}\right) \eta_{r v}^{(u),(i)}
$$

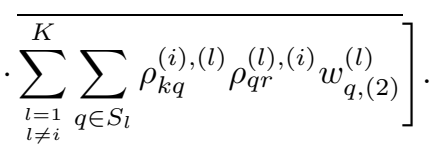

For the system parameters in Fig. 3, we found the optimum weights computed through closed-form expressions in (37) and (38) to be consistent with the weights for which maximum SIRs occur in Fig. 3.

\section{REFERENCES}

[1] K. Kim, Y. Han, and S.-L. Kim, "Joint subcarrier and power allocation in uplink OFDMA systems," IEEE Commun. Lett., vol. 9, no. 6, pp. 526-528, June 2005.

[2] Z. R. Cao, U. Tureli, and Y.-D. Yao, "Deterministic multiuser carrier frequency offset estimation for interleaved OFDMA uplink," IEEE Trans. Commun., vol. 52, no. 9, pp. 1585-1594, Sep. 2004.

[3] H. Wang and B. Chen, "Asymptotic distributions and peak power analysis for uplink OFDMA," in Proc. IEEE ICASSP, May 2004, pp. iv-1085-8.

[4] M. O. Pun, C.-C. J. Juo, and M. Morelli, "Joint synchronization and channel estimation in uplink OFDMA systems," in Proc. IEEE ICASSP, Mar. 2005, vol. 3, pp. iii/857-iii/860.

[5] A. M. Tonello, N. Laurenti, and S. Pupolin, "Analysis of the uplink of an asynchronous multiuser DMT OFDMA system impaired by time offsets, frequency offsets, and multipath fading," in Proc. IEEE VTC (Fall), Oct. 2000, vol. 3, pp. 1094-1099. 
[6] J. Choi, C. Lee, H. W. Jung, and Y. H. Lee, "Carrier frequency offset compensation for uplink of OFDM-FDMA systems," IEEE Commun. Lett., vol. 4, no. 12, pp. 414-416, Dec. 2000.

[7] Z. Cao, U. Tureh, and Y. D. Yao, "Analysis of two receiver schemes for interleaved OFDMA uplink signals," in Proc. 36th Asilomar Conf. Signals, Syst. Comput., Nov. 2002, vol. 2, pp. 1818-1821.

[8] R. Fantacci, D. Marabissi, and S. Papini, "Multiuser interference cancellation receivers for OFDMA uplink communications with carrier frequency offset," in Proc. IEEE GLOBECOM, Nov.-Dec. 2004, pp. 2808-2812.

[9] D. Huang and K. B. Letaief, "An interference cancellation scheme for carrier frequency offsets correction in OFDMA systems," IEEE Trans. Commun., vol. 53, no. 7, pp. 1155-1165, July 2005.

[10] D. Sreedhar and A. Chockalingam, "MMSE receiver for multiuser interference cancellation in uplink OFDMA," in Proc. IEEE VTC (Spring), May 2006, pp. 2125-2129.

[11] T. Pollet, M. V. Bladel, and M. Moeneclaey, "BER sensitivity of OFDM systems to carrier frequency offset and Weiner phase noise," IEEE Trans. Commun., vol. 43, pp. 191-193, Feb./Mar./Apr. 1995.

[12] L. Rugini, P. Banelli, and S. Cacopardi, "Probability of error of OFDM systems with carrier offset in frequency-selective fading channels," in Proc. IEEE GLOBECOM, Nov.-Dec. 2004, pp. 3289-3293.

[13] M. Varanasi and B. Aazhang, "Multistage detection in asynchronous code-division multiple-access," IEEE Trans. Commun., vol. 38, pp. 509519, Apr. 1990.

[14] D. Divsalar, M. K. Simon, and D. Raphaeli, "Improved parallel interference cancellation for CDMA," IEEE Trans. Commun., vol. 46, no. 2, pp. 258-268, Feb. 1998.

[15] D. R. Brown, M. Motani, V. Veeravalli, H. V. Poor, and C. R. Johnson, Jr., "On the performance of linear parallel interference cancellation," IEEE Trans. Inform. Theory, vol. 47, no. 5, pp. 1957-1970, July 2001

[16] D. Guo, L. K. Rasmussen, S. Sun, and T. J. Lim, "A matrix-algebraic approach to linear parallel interference cancellation in CDMA," IEEE Trans. Commun., vol. 48, no. 1, pp. 152-161, Jan. 2000.

[17] Y.-H. Li, M. Chen, and S.-X. Cheng, "Determination of cancellation factors for soft decision partial PIC detector in DS-CDMA systems," IEEE Electron. Lett., vol. 36, no. 3, pp. 239-241, Feb. 2000.

[18] V. Tikiya, S. Manohar, and A. Chockalingam, "SIR-optimized weighted linear parallel interference canceller on fading channels," IEEE Trans. Wireless Commun., vol. 5, no. 8, pp. 1998-2003, Aug. 2006.

[19] IEEE Standard for Local and Metropolitan Area Networks. Part 16: Air Interface for Fixed and Mobile Broadband Wireless Access Systems. Amendment 2: Physical and Medium Access Control Layers for Combined Fixed and Mobile Operation in Licensed Bands, IEEE Standard 802.16e-2005, 2005.

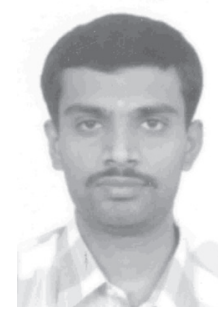

Shamaiah Manohar received his Master of Engineering degree in Electrical Communication Engineering from the Indian Institute of Science, Bangalore, India, in 2005, and the Bachelor of Engineering degree in Electronics and Communication Engineering from the Visveswaraiah Technological University, Belgaum, Karnataka, India, in 2003. He is currently working with Honeywell Technology Solutions Lab Private Limited, Bangalore, India, as a senior engineer. His research interests include UWB, CDMA and OFDM systems.

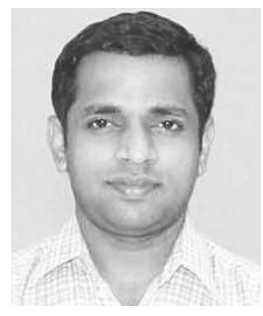

Dheeraj Sreedhar was born in Calicut, India in the year 1975. He received his B.Tech degree from the Indian Institute of Technology Madras in the year 1997, and his Master of Engineering degree from the Indian Institute of Science, Bangalore in the year 1999. Since 1999, he has been with Sasken Communication Technologies Limited, Bangalore as a technical architect. He is currently working towards his Ph.D degree in the Department of Electrical and Communication Engineering at the Indian Institute of Science, Bangalore. His current research interests include wireless communication systems and source coding.

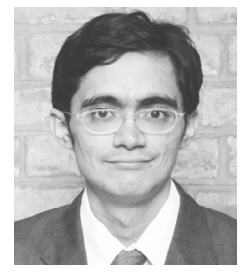

Vibhor Tikiya received his Master of Engineering degree in Electrical Communication Engineering from the Indian Institute of Science, Bangalore, India, in 2004, and the Bachelor of Engineering degree from the Mumbai University, India, in 2002. $\mathrm{He}$ is currently a student at the Indian Institute of Management, Ahmedabad, India. His research interests are in the area of wireless communications and pricing in telecommunication networks.

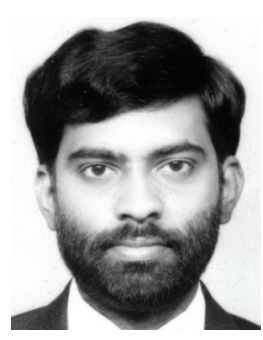

A. Chockalingam received the B.E. (Honors) degree in Electronics and Communication Engineering from the P. S. G. College of Technology, Coimbatore, India, in 1984, the M.Tech. degree with specialization in satellite communications from the Indian Institute of Technology, Kharagpur, India, in 1985, and the Ph.D. degree in Electrical Communication Engineering (ECE) from the Indian Institute of Science (IISc), Bangalore, India, in 1993. During 1986 to 1993 , he worked with the Transmission R \& D division of the Indian Telephone Industries Ltd., Bangalore. From December 1993 to May 1996, he was a Postdoctoral Fellow and an Assistant Project Scientist at the Department of Electrical and Computer Engineering, University of California, San Diego (UCSD). From May 1996 to December 1998, he served Qualcomm, Inc., San Diego, CA, as a Staff Engineer/Manager in the systems engineering group. In December 1998, he joined the faculty of the Department of ECE, IISc, Bangalore, India, where he is an Associate Professor, working in the area of wireless communications and networking. He was a visiting faculty to UCSD during summers of 1999-2002. He is a recipient of the Swarnajayanti Fellowship from the Department of Science and Technology, Government of India. He currently serves as an Associate Editor for the IEEE TRANSACTIONS ON VehicUlar TECHNOLOGY and the IEEE TRANSACTIONS ON WirElESS Communications. He is a Fellow of the Indian National Academy of Engineering. 